\title{
284 INTEGRATED MOLECULAR CHARACTERIZATION OF PRIMARY RESISTANCE MECHANISMS TO IMMUNE CHECKPOINT BLOCKADE IN ADVANCED NON-SMALL CELL LUNG CARCINOMA (A-NSCLC)
}

${ }^{1}$ Benjamin Besse, ${ }^{2} \mathrm{DIB}$ Colette, ${ }^{2}$ Eladio Marquez*, ${ }^{2}$ Joon Sang Lee, ${ }^{2}$ Shu Yan ${ }^{2}$ Marielle Chiron, ${ }^{2}$ Cecile Combeau, ${ }^{2}$ Angelique Biancotto, ${ }^{1}$ Félix Blanc-Durand, ${ }^{1}$ Mihaela Aldea, ${ }^{1}$ David Planchard, ${ }^{1}$ Jean-Yves Scoazec, 'Ludovic Lacroix, ${ }^{1}$ Etienne Rouleau, ${ }^{1}$ Nathalie Chaput-Gras, ${ }^{1}$ Saloomeh Rafie, ${ }^{1}$ Aurélien Marabelle, ${ }^{1}$ Eric Angevin, ${ }^{2}$ Jack Pollard. ${ }^{1} / G R$, Villejuif, France; ${ }^{2}$ Sanofi, VITRY SUR SEINE, France

Background Reinvigoration of anti-tumor immunity via immune checkpoint blockade (ICB) has transformed outcomes in a-NSCLC. However, a majority of patients are innately resistant to $\mathrm{ICB}$, and a better understanding of the resistance mechanisms may guide the development of new treatment strategies and therapies for patients.

Methods Biopsies performed immediately before treatment with single agent ICB in patients with a-NSCLC (MATCH-R trial [NCT02517892]) were analyzed. The stromal microenvironment and immune context were characterized via an integrated analysis of whole transcriptome (RNA-seq), whole exome sequencing (WES), and immunohistochemistry (IHC) of CD3, CD8, FOXP3 and PDL1. Specifically, the immune context and the relative abundance of 10 immune and stromal cell types were assessed with integrated IHC and Cell Populations-counter (MCP-counter) [1] analysis of the RNA-seq. Somatic mutations and Tumor Mutation Burden (TMB) were evaluated. The transcriptional state of the tumor and its microenvironment were assessed by GSVA analysis [2] of the MSigDB collection [3]. Patient's outcome was associated to molecular data. Primary resistance to ICB was defined as PD (progressive disease) in the first radiological examination, or a median PFS inferior to 3 months.

Results Fifty-two patients with NSCLC were enrolled (43 adeno, 6 squamous, and 3 other carcinoma): Median age was 61 (34-93), 18 were female, 46 were smokers, 22 were responders, and 30 were non-responders. Median tumor cellularity was 60\% (30\%-90\%).Patients may be divided into two groups (HIGH and LOW) at baseline based on their degree of immune infiltration as assessed by RNAseq or IHC. A hallmark of the HIGH infiltration group is an increase in Interferon Gamma (IFN- $\gamma$ ) pathway signature [4]. In contrast, patients in the LOW infiltration group (relative to the HIGH infiltration group) exhibit a decrease in IFN- $\gamma$ pathway signaling and concomitantly an increase in hypoxia and gluconeogenic pathway signatures. Response rates to ICB were not associated to immune infiltration groups at baseline, but an analysis within each infiltration group revealed that high TMB is only associated to response in the HIGH infiltration group. Furthermore, only in the LOW infiltration group was increased the transforming growth factor (TGF- $\beta$ ) pathway signature associated to ICB response.

Conclusions This study suggests that the tumor and its microenvironment influence baseline immune infiltration. Tumors with LOW baseline infiltration show altered metabolism such as gluconeogenic activation and hypoxia activation. In contrast, factors such as TMB are not associated with baseline infiltration

http://dx.doi.org/10.1136/jitc-2021-SITC2021.284 